\title{
Coarse base change fails for some modular curves
}

\author{
Kęstutis Česnavičius
}

\begin{abstract}
For a congruence level $H \subset \mathrm{GL}_{2}(\widehat{\mathbb{Z}})$, the formation of the modular curve $X_{H}$, that is, of the coarse moduli space of the level $H$ modular stack $\mathscr{X}_{H}$, is known to commute with arbitrary base change in a wide range of cases. We exhibit infinitely many $H$, for instance $H=\Gamma_{1}(4)$, for which this coarse base change property fails. In our examples, failure is witnessed for base change to $\mathbb{F}_{2}$ and for any $\mathbb{Z}_{(2)}$-fiberwise dense open substack of $\mathscr{X}_{H}$. These examples fill in several open entries in a table in the book of Katz and Mazur.
\end{abstract}

\section{Introduction}

1.1. Coarse base change for modular curves. In the study of modular forms modulo a prime $p$, one often considers the $\mathbb{F}_{p}$-fiber $\left(X_{H}\right)_{\mathbb{F}_{p}}$ of the modular curve $X_{H}$ over $\mathbb{Z}$ for an open subgroup $H \subset \mathrm{GL}_{2}(\widehat{\mathbb{Z}})$. This $\mathbb{F}_{p}$-fiber may be difficult to analyze $-X_{H}$ is defined as the coarse moduli space of the level $H$ modular stack $\mathscr{X}_{H}$, but there is no a priori reason why $\left(X_{H}\right)_{\mathbb{F}_{p}}$ should be the coarse space of $\left(\mathscr{X}_{H}\right)_{\mathbb{F}_{p}}$ if $p=2$ or $p=3$. If, however, $\left(X_{H}\right)_{\mathbb{F}_{p}}$ happens to be the coarse space of $\left(\mathscr{X}_{H}\right)_{\mathbb{F}_{p}}$ for every prime $p$, then one knows (say, from [Čes15, Lemma 3.3.1]) that coarse base change holds for $\mathscr{X}_{H}$, that is, that $\left(X_{H}\right)_{S}$ is the coarse space of $\left(\mathscr{X}_{H}\right)_{S}$ for every scheme $S$. For instance, this is the case when $H=\mathrm{GL}_{2}(\widehat{\mathbb{Z}})$. The main goal of this paper is to show that coarse base change fails for some $\mathscr{X}_{H}$.

1.2. Known results on coarse base change for modular curves. To put our examples in perspective, we turn to known results, all of which confirm coarse base change for $\mathscr{X}_{H}$ for suitable $H$.

Only primes 2 and 3 may divide the order of the automorphism group of an elliptic curve over a field, so it is a generality that $\left(X_{H}\right)_{S}$ is the coarse moduli space of $\left(\mathscr{X}_{H}\right)_{S}$ whenever $S$ is a $\mathbb{Z}\left[\frac{1}{6}\right]$-scheme (the essential observation is that the formation of the ring of invariants $R^{G}$ for the action of a finite group $G$ on a ring $R$ commutes with arbitrary base change whenever $\# G \in R^{\times}$). As is explained in [Čes15, Proposition 6.4(b)], it then follows from the results of Deligne-Rapoport and Katz-Mazur that the same holds whenever $S$ is a $\mathbb{Z}[1 / \operatorname{gcd}(6, n)]$-scheme for any $n \in \mathbb{Z}_{\geqslant 1}$ with $\Gamma(n) \subset H$, where $\Gamma(n):=\operatorname{Ker}\left(\mathrm{GL}_{2}(\widehat{\mathbb{Z}}) \rightarrow \mathrm{GL}_{2}(\mathbb{Z} / n \mathbb{Z})\right)$.

There are also situations in which 2 or 3 divides the level and coarse base change continues to hold (with $\mathscr{X}_{H} \neq X_{H}$ ). For instance, by [DR73, VI, Corollaire 6.10] (with [Čes15, Lemma 3.3.1]

Received 1 May 2016, accepted in final form 22 October 2016.

2010 Mathematics Subject Classification 11G18 (primary), 14D22, 14D23, 14G35 (secondary).

Keywords: base change, coarse moduli space, level structure, modular curve.

This journal is (C) Foundation Compositio Mathematica 2017. This article is distributed with Open Access under the terms of the Creative Commons Attribution Non-Commercial License, which permits non-commercial reuse, distribution, and reproduction in any medium, provided that the original work is properly cited. For commercial re-use, please contact the Foundation Compositio Mathematica. 


\section{COARSE BASE CHANGE FAILS FOR SOME MODUlar CURVES}

as before), this is the case when $H=\Gamma_{0}(6) \cap H^{\prime}$ with an $H^{\prime}$ for which $\Gamma(n) \subset H^{\prime}$ for some $n \in \mathbb{Z}_{\geqslant 1}$ that is prime to 6 .

One may also ask whether coarse base change holds generically, say, whether it holds for the open substack $\mathscr{U} \subset \mathscr{X}_{H}$ on which the $j$-invariant avoids the set $\{0,1728, \infty\}$ (this automatically disposes of the need to consider the $\mathbb{F}_{3}$-fiber). To address this, Katz and Mazur compiled a table $[K M 85,8.5 .4]$ that confirms coarse base change for $\mathscr{U}$ for many common choices of $H$. The examples of this paper fill in a "NO" for some open entries of this table.

1.3. The nature of our examples. We choose the subgroup $H$ to be either

$$
H=\Gamma_{1}(4) \cap \Gamma_{0}(4 n) \quad \text { for an } n \in \mathbb{Z}_{\geqslant 1} \quad \text { or } \quad H=\Gamma_{1}^{\text {bal }}(4)
$$

(see $\S \S 3-5$ for a review of the definitions of these subgroups) and show that $\left(X_{H}\right)_{\mathbb{F}_{2}}$ is not the coarse moduli space of $\left(\mathscr{X}_{H}\right)_{\mathbb{F}_{2}}$. For the sake of clarity, we first treat the simplest case $H=\Gamma_{1}(4)$ in $\S 3$ and then refine the argument in $\S 4$ to include all $H$ of the form $\Gamma_{1}(4) \cap \Gamma_{0}(4 n)$. This family of examples shows that coarse base change may fail for modular curves of arbitrarily high genus.

In our examples, the failure of coarse base change persists to fiberwise dense open substacks (such as $\mathscr{U}$ of $\S 1.2$ ) and results from the jumping of generic stabilizers: for $H$ as in (1.1), the stack $\left(\mathscr{X}_{H}\right)_{\mathbb{Q}}$ is a scheme, whereas some open substack of $\left(\mathscr{X}_{H}\right)_{\mathbb{F}_{2}}$ is a $\mathbb{Z} / 2 \mathbb{Z}$-gerbe over its coarse space. The conclusion then results from the fact that over a fiberwise dense open subset of $\left(X_{H}\right)_{\mathbb{Z}_{(2)}}$, the coarse moduli space morphism

$$
\left(\mathscr{X}_{H}\right)_{\mathbb{Z}_{(2)}} \rightarrow\left(X_{H}\right)_{\mathbb{Z}_{(2)}}
$$

is flat (and hence, morally, finite, locally free of rank 1), as is ensured by the miracle flatness theorem combined with an openness of the regular locus result of Nagata (in the form of [EGAIV ${ }_{2}$, Corollaire 6.12.6]); see $\S 2$, especially the proof of Theorem 2.1, for the details of this argument.

In view of the results of this paper, the remaining open entries in the table [KM85, 8.5.4] are those of $\left[\Gamma_{1}(N)\right]$ with $N=8,16,32, \ldots$ and those of $\left[\Gamma_{0}\left(2^{n} ; a, b\right)\right]$ with $a \geqslant 3$ and $b=0$ (the assumption $a \geqslant b$ loses no generality and $\left.\left[\Gamma_{0}\left(2^{n} ; a, 1\right)\right]=\left[\Gamma_{0}\left(2^{n} ; a, 0\right)\right]\right)$.

It would be interesting to understand whether coarse base change may also fail at individual closed points. More precisely, it would be interesting to answer the following question.

QUESTION 1.4. Are there an open subgroup $H \subset \mathrm{GL}_{2}(\widehat{\mathbb{Z}})$ and a prime $p \in\{2,3\}$ such that coarse base change holds for some $\mathbb{Z}_{(p)}$-fiberwise dense open substack $\mathscr{V} \subset\left(\mathscr{X}_{H}\right)_{\mathbb{Z}_{(p)}}$ but fails for $\left(\mathscr{X}_{H}\right)_{\mathbb{Z}_{(p)}}$ itself? In particular, can coarse base change fail for $\left(\mathscr{X}_{H}\right)_{\mathbb{Z}_{(3)}}$ ?

1.5. Notation and conventions. For an open subgroup $H \subset \mathrm{GL}_{2}(\widehat{\mathbb{Z}})$, we let $\mathscr{X}_{H}$ denote the level $H$ Deligne-Mumford modular stack over $\mathbb{Z}$ defined in [DR73, IV, Définition 3.3] by normalization (we also rely on the agreement discussed in [Čes15, Proposition 6.3] with the definitions used in [KM85]). Replacement of $\mathscr{X}$ by $\mathscr{Y}$ - for instance, of $\mathscr{X}_{H}$ by $\mathscr{Y}_{H}$-indicates the elliptic curve locus. Level structures are in the sense of Drinfeld - for instance, a finite, locally free $S$-subgroup $G \subset E$ of an elliptic curve $E \rightarrow S$ is cyclic of order $n$ if locally on $S$ for the faithfully flat of finite presentation (fppf) topology, it admits a Drinfeld $\mathbb{Z} / n \mathbb{Z}$-structure (the needed definitions are reviewed in [Čes15, Definitions 4.2.2, 4.2.6, and 4.2.8]). For an $n \in \mathbb{Z}_{\geqslant 1}$, we let $\Gamma(n) \subset \mathrm{GL}_{2}(\widehat{\mathbb{Z}})$ be as in $\S 1.2$, and we write $\mathscr{X}(1)$ for $\mathscr{X}_{\Gamma(1)}$ (that is, for $\left.\mathscr{X}_{\mathrm{GL}_{2}(\widehat{\mathbb{Z}})}\right)$. For a prime $p$, we let $(-)_{(p)}$ denote localization at $p$. 


\section{K. ČEsnaviČIUS}

\section{The effect on coarse base change of the jumping of the generic stabilizer}

The following criterion is the source of our examples of the failure of coarse base change.

TheOREM 2.1. Suppose that $H \subset \mathrm{GL}_{2}(\widehat{\mathbb{Z}})$ is an open subgroup for which there exists an open substack

such that

$$
\mathscr{U} \subset\left(\mathscr{Y}_{H}\right)_{\mathbb{Z}_{(2)}}
$$

(i) the automorphism groups of the geometric points of $\mathscr{U}_{\mathbb{Q}}$ are trivial (that is, $\mathscr{U}_{\mathbb{Q}}$ is a scheme, cf. [Ces15, Remark 4.1.4]);

(ii) there is a nonempty open substack $\mathscr{U}^{\prime} \subset \mathscr{U}_{\mathbb{F}_{2}}$ whose inertia stack is the constant $\underline{\{ \pm 1\}_{\mathscr{U}^{\prime}}}$.

Then the base change

$$
\pi_{\mathbb{F}_{2}}:\left(\mathscr{X}_{H}\right)_{\mathbb{F}_{2}} \rightarrow\left(X_{H}\right)_{\mathbb{F}_{2}}
$$

of the coarse moduli space morphism $\pi: \mathscr{X}_{H} \rightarrow X_{H}$ is not the coarse moduli space morphism of $\left(\mathscr{X}_{H}\right)_{\mathbb{F}_{2}}$; in fact, for the coarse moduli space $U:=\pi_{\mathbb{Z}_{(2)}}(\mathscr{U})$ of $\mathscr{U}$, the map

$$
\left.\left(\pi_{\mathbb{F}_{2}}\right)\right|_{\mathscr{U}_{\mathbb{F}_{2}}}: \mathscr{U}_{\mathbb{F}_{2}} \rightarrow U_{\mathbb{F}_{2}}
$$

is not a coarse moduli space morphism.

Remark 2.2. In practice, we will have $\mathscr{U}=\left(\mathscr{Y}_{H}\right)_{\mathbb{Z}_{(2)}}$. However, Theorem 2.1 with an arbitrary $\mathscr{U}$ includes the claim that in presence of conditions (i) and (ii), the failure of coarse base change for $\mathscr{X}_{H}$ cannot be remedied by removing a $\mathbb{Z}$-quasi-finite closed substack of $\mathscr{X}_{H}$ (such as the preimage of the sections $j=0, j=1728$, and $j=\infty$ of the $j$-line $\mathbb{P}_{\mathbb{Z}}^{1}$ ).

Proof. We replace $\mathscr{U}$ by $\mathscr{U} \backslash\left(\mathscr{U}_{\mathbb{F}_{2}} \backslash \mathscr{U}^{\prime}\right)$ to be able to assume $\mathscr{U}^{\prime}=\mathscr{U}_{\mathbb{F}_{2}}$. Also, it suffices to establish the last claim, so we may work over $U$ and, in particular, within the elliptic curve locus.

The flat relative $\mathbb{Z}_{(2)}$-curve $U$ inherits normality from $\left(X_{H}\right)_{\mathbb{Z}_{(2)}}$, so, by $[$ EGAIV 2 , Corollaire 6.12.6], the curve $U$ is regular away from finitely many closed points of residue characteristic 2 . We remove these points from $U$ and remove their preimages from $\mathscr{U}$ to assume for the rest of the proof that $U$ is regular.

Let $n \in \mathbb{Z}_{\geqslant 1}$ be such that $\Gamma(n) \subset H$, let $p$ be an odd prime that does not divide $n$, and consider $\mathscr{Y}_{H \cap \Gamma(p)}$. The $\mathbb{Z}_{(2)}$-base change $\left(\mathscr{Y}_{H \cap \Gamma(p)}\right)_{\mathbb{Z}_{(2)}}$ is a scheme because the same holds already for $\left(\mathscr{Y}_{\Gamma(p)}\right)_{\mathbb{Z}_{(2)}}$ (cf. [DR73, IV, Corollaire 2.9]). By [DR73, IV, proof of Proposition 3.9], ${ }^{1}$

$$
\mathscr{Y}_{H \cap \Gamma(p)}=\mathscr{Y}_{H} \times \mathscr{Y}(1) \mathscr{Y}_{\Gamma(p)},
$$

so, by [DR73, IV, paragraph after Définition 2.4],

$$
\left(\mathscr{Y}_{H \cap \Gamma(p)}\right)_{\mathbb{Z}_{(2)}} \rightarrow\left(\mathscr{Y}_{H}\right)_{\mathbb{Z}_{(2)}} \text { is a } \mathrm{GL}_{2}(\mathbb{Z} / p \mathbb{Z}) \text {-torsor, }
$$

and hence is finite étale of degree $\# \mathrm{GL}_{2}(\mathbb{Z} / p \mathbb{Z})$. Moreover, if

$$
\mathscr{V} \subset\left(\mathscr{Y}_{H \cap \Gamma(p)}\right)_{\mathbb{Z}_{(2)}}
$$

denotes the preimage of $\mathscr{U} \subset\left(\mathscr{Y}_{H}\right)_{\mathbb{Z}_{(2)}}$ (equivalently, of $U$ ), then the regularity of $U$ and the miracle flatness theorem $\left[\mathrm{EGAIV}_{2}\right.$, Proposition 6.1.5] ensure that the finite morphism $\mathscr{V} \rightarrow U$ is locally free. Due to the $\mathbb{Z}_{(2)}$-flatness of $U$, the degree of this morphism may be read off over $\mathbb{Q}$,

\footnotetext{
${ }^{1}$ Even though the statement of [DR73, IV, Proposition 3.9] is incorrect (see [Čes15, Example 4.5.3] for a counterexample), its proof justifies the analogous claim over the elliptic curve locus.
} 


\section{COARSE BASE CHANGE FAILS FOR SOME MODUlAR CURVES}

and hence, thanks to condition (i) and (2.1), equals \# $\mathrm{GL}_{2}(\mathbb{Z} / p \mathbb{Z})$. For the sought conclusion, we will show that, in contrast, the map

$$
\mathscr{V}_{\mathbb{F}_{2}} \rightarrow U^{\prime}
$$

toward the coarse moduli space $U^{\prime}$ of $\mathscr{U}_{\mathbb{F}_{2}}$ is finite, locally free of degree $\frac{1}{2} \cdot \# \mathrm{GL}_{2}(\mathbb{Z} / p \mathbb{Z})$.

Due to condition (ii), the space $U^{\prime}$ identifies with the rigidification $\mathscr{U}^{\prime} \mathbb{Z}\{ \pm 1\}$ (cf. [AOV08, Theorem A.1] or [Rom05, Theorem 5.1], as well as [LM00, Corollaire 8.1.1]), so $\mathscr{V}_{\mathbb{F}_{2}} \rightarrow U^{\prime}$ is finite, locally free. To verify that twice its degree equals the claimed $\# \mathrm{GL}_{2}(\mathbb{Z} / p \mathbb{Z})$, it remains to work étale locally on $U^{\prime}$ to first obtain an isomorphism

$$
\mathscr{U}^{\prime} \simeq \mathbf{B}\left(\underline{\mathbb{Z} / 2 \mathbb{Z}}_{U^{\prime}}\right)
$$

with a classifying stack and to then base change the resulting $\mathrm{GL}_{2}(\mathbb{Z} / p \mathbb{Z})$-torsor

$$
\mathscr{V}_{\mathbb{F}_{2}} \rightarrow \mathbf{B}\left(\underline{\mathbb{Z} / 2 \mathbb{Z}}_{U^{\prime}}\right)
$$

along the étale atlas $U^{\prime} \rightarrow \mathbf{B}\left(\underline{\mathbb{Z} / 2 \mathbb{Z}} U_{U^{\prime}}\right)$ that corresponds to the trivial $\underline{\mathbb{Z} / 2 \mathbb{Z}} U_{U^{\prime}}$-torsor.

Remark 2.3. The proof shows that the comparison morphism from the coarse moduli space of $\mathscr{U}^{\prime}$ toward $U_{\mathbb{F}_{2}} \cap \pi_{\mathbb{F}_{2}}\left(\mathscr{U}^{\prime}\right)$ is finite, locally free of rank 2 over a dense open subscheme of $U_{\mathbb{F}_{2}} \cap \pi_{\mathbb{F}_{2}}\left(\mathscr{U}^{\prime}\right)$. It is a generality that this morphism is necessarily a universal homeomorphism (cf. [Ryd13, Theorem 6.12]).

For $H$ discussed below, the construction of suitable open substacks $\mathscr{U}^{\prime}$ rests on the following known lemmas.

Lemma 2.4 ([Del75, Proposition 5.3(III)]). If $S$ is a scheme and $E \rightarrow S$ is an elliptic curve whose fibral $j$-invariants differ from 0 and 1728, then the automorphism functor of $E$ is the constant $\{ \pm 1\}_{S}$.

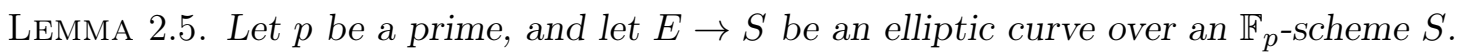

(a) ([KM85, Lemma 12.2.5]). For every $n \in \mathbb{Z}_{\geqslant 1}$, the $S$-subgroup $E\left[p^{n}\right] \subset E$ is cyclic of order $p^{2 n}$, and its standard cyclic subgroup of order $p^{n}$ is the kernel of the $n$-fold relative Frobenius of $E \rightarrow S$.

(b) If $p=2$ and $G \subset E$ is a cyclic $S$-subgroup of order $p^{2}$ such that its standard cyclic subgroup $G_{p}$ of order $p$ is of multiplicative type and $G / G_{p}$ is étale, then $G=E[p]$.

Proof. The indicated reference supplies part (a). For part (b), we may use limit arguments to assume that $S$ is connected, so [KM85, Theorem 13.3.3] shows that $G$ agrees with the kernel of the composite isogeny

$$
E \stackrel{\operatorname{Frob}_{E}}{\longrightarrow} E^{(p)} \stackrel{\iota}{\sim} \widetilde{E}^{(p)} \stackrel{\operatorname{Ver}_{\widetilde{E}}}{\longrightarrow} \widetilde{E}
$$

for some elliptic curve $\widetilde{E} \rightarrow S$ and some $S$-isomorphism $\iota$. By Lemma 2.4, any $S$-automorphism of $E^{(p)}$ is multiplication by \pm 1 , so it remains to show that $E \simeq \widetilde{E}$ étale locally on $S$, which follows from [KM85, Theorem 13.3.5(4)] after endowing $E$ and $\widetilde{E}$ with compatible (étale local on $S$ ) $(\mathbb{Z} / 3 \mathbb{Z})^{2}$-structures (the $p=2$ assumption ensures that the $(p-1)$ st infinitesimal neighborhood of the diagonal in loc. cit. coincides with the diagonal itself). 


\section{K. ČesnaviČIUS}

\section{Coarse base change fails for $\mathscr{X}_{1}(4)$}

In this section, we choose the level $H$ to be

$$
\Gamma_{1}(4)=\left\{\left(\begin{array}{ll}
a & b \\
c & d
\end{array}\right) \in \mathrm{GL}_{2}(\widehat{\mathbb{Z}}) \mid a \equiv 1 \bmod 4, c \equiv 0 \bmod 4\right\}
$$

and seek to show that the formation of the coarse moduli space of $\mathscr{X}_{1}(4):=\mathscr{X}_{\Gamma_{1}(4)}$ does not commute with base change to $\mathbb{F}_{2}$. We will obtain this from Theorem 2.1, so we first explain how we choose the open substack

$$
\mathscr{U}^{\prime} \subset \mathscr{Y}_{1}(4)_{\mathbb{F}_{2}}
$$

of the elliptic curve locus over $\mathbb{F}_{2}$ (we will choose $\mathscr{U}$ to be $\mathscr{Y}_{1}(4)_{\mathbb{Z}_{(2)}}$ ).

3.1. The choice of $\mathscr{U}^{\prime}$. By [KM85, 3.2] (see also [Čes15, Theorem 4.4.4(c)]), the DeligneMumford stack $\mathscr{Y}_{1}(4)$ parametrizes pairs

$$
(E \rightarrow S, \alpha: \mathbb{Z} / 4 \mathbb{Z} \rightarrow E(S))
$$

consisting of an elliptic curve $E \rightarrow S$ over a variable base scheme $S$ and a Drinfeld $\mathbb{Z} / 4 \mathbb{Z}$ structure $\alpha$ on $E$. Consider the stack $\mathscr{Y}_{1}(4)_{\mathbb{F}_{2}}$ together with its universal elliptic curve $\mathcal{E} \rightarrow \mathscr{Y}_{1}(4)_{\mathbb{F}_{2}}$, and let $\mathcal{G} \subset \mathcal{E}$ be the finite, locally free $\mathscr{Y}_{1}(4)_{\mathbb{F}_{2}}$-subgroup of order 4 generated by the universal $\alpha$. The locus $\mathscr{U}^{\prime \prime}$ of $\mathscr{Y}_{1}(4)_{\mathbb{F}_{2}}$ over which $\mathcal{G}$ is of multiplicative type is open, and we let

$$
\mathscr{U}^{\prime} \subset \mathscr{U}^{\prime \prime}
$$

be the open locus on which the $j$-invariant satisfies $j \neq 0$.

TheOREM 3.2. The $\mathbb{F}_{2}$-base change of the coarse space morphism of $\mathscr{X}_{1}(4)$ is not the coarse space morphism of $\mathscr{X}_{1}(4)_{\mathbb{F}_{2}}$. The same holds for any $\mathbb{Z}_{(2)}$-fiberwise dense open substack of $\mathscr{X}_{1}(4)$.

Proof. The claim will follow from Theorem 2.1 once we explain why

$$
\mathscr{U}:=\mathscr{Y}_{1}(4)_{\mathbb{Z}_{(2)}} \quad \text { and } \quad \mathscr{U}^{\prime} \text { of Section } 3.1
$$

satisfy its assumptions (i) and (ii). The assumption (i) follows from the rigidity of $\mathscr{Y}_{1}(4)_{\mathbb{Z}\left[\frac{1}{2}\right]}$, that is, from [KM85, Corollary 2.7.4 and Lemma 1.5.3]. For assumption (ii), we start with the nonemptiness of $\mathscr{U}^{\prime}$, which follows from that of $\mathscr{U}^{\prime \prime}$ guaranteed by the fact that the ordinary locus of $\mathscr{Y}(1)_{\mathbb{F}_{2}}$ is the forgetful image of $\mathscr{U}^{\prime \prime}$ (cf. Lemma 2.5(a)).

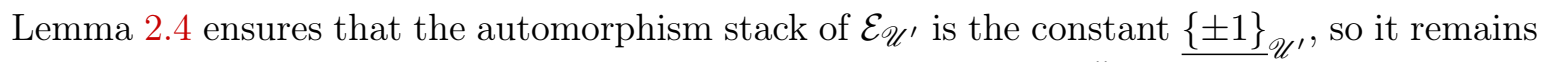
to show that $[-1]_{E}$ preserves $\alpha$ for every $(E \rightarrow S, \alpha)$ classified by $\mathscr{U}^{\prime \prime}$, that is, that $2 \cdot \alpha(1)=0$ for every such $(E, \alpha)$. For this, if $G$ denotes the finite, locally free subgroup of order 4 generated by $\alpha$ and $G_{2} \subset G$ denotes its standard cyclic subgroup of order 2, then it suffices to show that the image of $\alpha(1)$ in $G / G_{2}$ vanishes. However, by [KM85, Theorem 6.7.4], this image generates $G / G_{2}$, so it remains to recall that, by [KM85, Theorem 6.1.1], the subscheme of generators

$$
\left(G / G_{2}\right)^{\times} \subset G / G_{2}
$$

is finite, locally free of rank 1 and that, by [KM85, Lemma 12.2.1 and its proof], the subscheme $\left(G / G_{2}\right)^{\times}$contains the zero section of $G / G_{2}$ because $G / G_{2}$ is the Frobenius kernel of $E / G_{2}$.

Remark 3.3. We could have analogously constructed a larger $\mathscr{U}^{\prime}$ by allowing $\mathscr{U}^{\prime \prime}$ to also contain the open locus of $\mathscr{Y}_{1}(4)_{\mathbb{F}_{2}}$ over which the standard cyclic subgroup $\mathcal{G}_{2} \subset \mathcal{G}$ of order 2 is of multiplicative type and $\mathcal{G} / \mathcal{G}_{2}$ is étale. Over this locus, $\mathcal{G}=\mathcal{E}[2]$ by Lemma $2.5(\mathrm{~b})$, so $2 \cdot \alpha(1)=0$, too. 


\section{COARSE BASE CHANGE FAILS FOR SOME MODUlAR CURVES}

Remark 3.4. By combining Remarks 2.3 and 3.3, we conclude that the comparison morphism from the coarse moduli space of $\mathscr{Y}_{1}(4)_{\mathbb{F}_{2}}$ to $Y_{1}(4)_{\mathbb{F}_{2}}$ is finite, locally free of rank 2 over a dense open subset of the locus over which $\mathcal{G}$ has a nontrivial multiplicative part and is a universal homeomorphism over the entire $Y_{1}(4)_{\mathbb{F}_{2}}$. In contrast, this morphism is an isomorphism over the locus over which $\mathcal{G}$ is étale because there $\mathscr{Y}_{1}(4)$ is rigid by [KM85, Corollary 2.7.4 and Proposition 1.10.12].

\section{Coarse base change fails for $\mathscr{X}_{\Gamma_{1}(4) \cap \Gamma_{0}(4 n)}$}

We seek to explain how a variant of the construction of $\S 3$ leads to an infinite family of modular curves that violate coarse base change. We fix any (possibly even) integer $n \in \mathbb{Z}_{\geqslant 1}$, set

$$
\Gamma_{0}(4 n):=\left\{\left(\begin{array}{ll}
a & b \\
c & d
\end{array}\right) \in \mathrm{GL}_{2}(\widehat{\mathbb{Z}}) \mid c \equiv 0 \bmod 4 n\right\},
$$

and seek to show that the formation of the coarse moduli space of $\mathscr{X}_{\Gamma_{1}(4) \cap \Gamma_{0}(4 n)}$ does not commute with base change to $\mathbb{F}_{2}$. The genus of the coarse moduli space of $\left(\mathscr{X}_{\Gamma_{1}(4) \cap \Gamma_{0}(4 n)}\right) \mathbb{Q}$ grows unboundedly with $n$ because the same holds already for the coarse moduli space of $\left(\mathscr{X}_{\Gamma_{0}(4 n)}\right)_{\mathbb{Q}}$.

As in $\S 3$, we first describe $\mathscr{Y}_{\Gamma_{1}(4) \cap \Gamma_{0}(4 n)}$ in modular terms and explain how we choose $\mathscr{U}^{\prime}$.

4.1. The choice of $\mathscr{U}^{\prime}$. By [KM85, Definition 7.9.4 and Theorems 7.9.6 and 7.4.2] (with [Čes15, Proposition 6.3(a)]), ${ }^{2}$ the stack $\mathscr{Y}_{\Gamma_{1}(4) \cap \Gamma_{0}(4 n)}$ parametrizes triples

$$
\left(E \rightarrow S, G, \alpha: \mathbb{Z} / 4 \mathbb{Z} \rightarrow\left(E / G_{n}\right)(S)\right)
$$

consisting of an elliptic curve $E \rightarrow S$ over a variable base scheme $S$, a cyclic $S$-subgroup $G \subset E$ of order $4 n$, and a Drinfeld $\mathbb{Z} / 4 \mathbb{Z}$-structure $\alpha$ on the $S$-subgroup $G / G_{n} \subset E / G_{n}$, where $G_{n} \subset G$ is the standard cyclic subgroup of order $n$.

We let

$$
\mathcal{E} \rightarrow\left(\mathscr{Y}_{\Gamma_{1}(4) \cap \Gamma_{0}(4 n)}\right)_{\mathbb{F}_{2}}
$$

be the universal elliptic curve in characteristic 2 and let $\mathcal{G} \subset \mathcal{E}$ be the universal $G$. The locus $\mathscr{U}^{\prime \prime}$ of $\left(\mathscr{Y}_{\Gamma_{1}(4) \cap \Gamma_{0}(4 n)}\right)_{\mathbb{F}_{2}}$ over which $\mathcal{G}$ is of multiplicative type is open, and we let

$$
\mathscr{U}^{\prime} \subset \mathscr{U}^{\prime \prime} \text { be the open locus on which the } j \text {-invariant satisfies } j \neq 0 \text {. }
$$

TheOREM 4.2. The $\mathbb{F}_{2}$-base change of the coarse space morphism of $\mathscr{X}_{\Gamma_{1}(4) \cap \Gamma_{0}(4 n)}$ is not the coarse space morphism of $\left(\mathscr{X}_{\Gamma_{1}(4) \cap \Gamma_{0}(4 n)}\right)_{\mathbb{F}_{2}}$. The same holds for any $\mathbb{Z}_{(2)}$-fiberwise dense open substack of $\mathscr{X}_{\Gamma_{1}(4) \cap \Gamma_{0}(4 n)}$.

Proof. Similarly to the proof of Theorem 3.2, it suffices to explain why Theorem 2.1 applies with

$$
\mathscr{U}:=\left(\mathscr{Y}_{\Gamma_{1}(4) \cap \Gamma_{0}(4 n)}\right)_{\mathbb{Z}_{(2)}} \quad \text { and } \quad \mathscr{U}^{\prime} \text { of } \S 4.1 .
$$

The validity of assumption (i) is again supplied by [KM85, Corollary 2.7.4].

For assumption (ii), the nonemptiness of the open substack $\mathscr{U}^{\prime \prime}$, and hence also of $\mathscr{U}^{\prime}$, follows from Lemma 2.5(a), which ensures that the ordinary locus of $\mathscr{Y}(1)_{\mathbb{F}_{2}}$ lies in the forgetful image of $\mathscr{U}^{\prime \prime}$. Thanks to Lemma 2.4, it remains to show that $2 \cdot \alpha(1)=0$ for every $(E, G, \alpha)$ classified by $\mathscr{U}^{\prime \prime}$. Since $G / G_{n} \subset E / G_{n}$ is of multiplicative type and cyclic of order 4 , this follows the same way as in the proof of Theorem 3.2.

\footnotetext{
${ }^{2}$ We implicitly also use [KM85, Propositions 1.7.2 and 7.3.1] to decompose into primary parts.
} 


\section{K. ČEsnaviČIUs}

\section{Coarse base change fails for $\mathscr{X}_{1}^{\text {bal }}(4)$}

In order to complete an open entry in the table [KM85, 8.5.4], we investigate coarse base change for one other level. More precisely, in this section we choose the level $H$ to be

$$
\Gamma_{1}^{\mathrm{bal}}(4):=\left\{\left(\begin{array}{ll}
a & b \\
c & d
\end{array}\right) \in \mathrm{GL}_{2}(\widehat{\mathbb{Z}}) \mid a, d \equiv 1 \bmod 4, c \equiv 0 \bmod 4\right\}
$$

and seek to show that the formation of the coarse moduli space of

$$
\mathscr{X}_{1}^{\mathrm{bal}}(4):=\mathscr{X}_{\Gamma_{1}^{\mathrm{bal}}(4)}
$$

does not commute with base change to $\mathbb{F}_{2}$. To again apply Theorem 2.1 , we first explain how we choose $\mathscr{U}^{\prime}$.

5.1. The choice of $\mathscr{U}^{\prime}$. By [KM85, 3.3] (with [KM85, Theorem 7.4.2(2)] and [Čes15, Proposition 6.3(a)]), the stack $\mathscr{Y}_{1}^{\text {bal }}(4)$ parametrizes triples

$$
\left(E \rightarrow S, \alpha: \mathbb{Z} / 4 \mathbb{Z} \rightarrow E(S), \beta: \mathbb{Z} / 4 \mathbb{Z} \rightarrow\left(E / G_{\alpha}\right)(S)\right)
$$

consisting of an elliptic curve $E \rightarrow S$ over a variable base scheme $S$, a Drinfeld $\mathbb{Z} / 4 \mathbb{Z}$-structure $\alpha$ on an $S$-subgroup $G_{\alpha} \subset E$, and a Drinfeld $\mathbb{Z} / 4 \mathbb{Z}$-structure $\beta$ on the $S$-subgroup $E[4] / G_{\alpha} \subset E / G_{\alpha}$.

Similarly to $\S 3.1$, we consider the universal elliptic curve

$$
\mathcal{E} \rightarrow \mathscr{Y}_{1}^{\mathrm{bal}}(4)_{\mathbb{F}_{2}}
$$

in characteristic 2 and let $\mathcal{G} \subset \mathcal{E}$ be the universal $G_{\alpha}$. The locus $\mathscr{U}^{\prime \prime}$ of $\mathscr{Y}_{1}^{\text {bal }}(4)_{\mathbb{F}_{2}}$ over which the standard cyclic subgroup $\mathcal{G}_{2} \subset \mathcal{G}$ of order 2 is of multiplicative type and $\mathcal{G} / \mathcal{G}_{2}$ is étale is open, and we let

$$
\mathscr{U}^{\prime} \subset \mathscr{U}^{\prime \prime} \text { be the open locus on which the } j \text {-invariant satisfies } j \neq 0 \text {. }
$$

TheOREM 5.2. The $\mathbb{F}_{2}$-base change of the coarse space morphism of $\mathscr{X}_{1}^{\mathrm{bal}}(4)$ is not the coarse space morphism of $\mathscr{X}_{1}^{\text {bal }}(4)_{\mathbb{F}_{2}}$. The same holds for any $\mathbb{Z}_{(2)}$-fiberwise dense open substack of $\mathscr{X}_{1}^{\mathrm{bal}}(4)$.

Proof. It suffices to explain why Theorem 2.1 applies with

$$
\mathscr{U}:=\mathscr{Y}_{1}^{\text {bal }}(4)_{\mathbb{Z}_{(2)}} \text { and } \quad \mathscr{U}^{\prime} \text { of } \S 5.1 .
$$

The assumption (i) follows from the rigidity of $\mathscr{Y}_{1}^{\text {bal }}(4)_{\mathbb{Z}\left[\frac{1}{2}\right]}$ supplied by [KM85, Corollary 2.7.4].

For assumption (ii), similarly to the proof of Theorem 3.2, we have to show that $\mathscr{U}^{\prime \prime} \neq \emptyset$ and that $\alpha(1)$ and $\beta(1)$ are 2 -torsion for every $(E, \alpha, \beta)$ classified by $\mathscr{U}^{\prime \prime}$. The latter requirement follows from Lemma $2.5(\mathrm{~b})$, which ensures that $\mathcal{G}_{\mathscr{U}^{\prime \prime}}=\mathcal{E}_{\mathscr{U}{ }^{\prime \prime}}[2]$. The nonemptiness, on the other hand, follows from Lemma 2.5(a), which ensures that the forgetful image of $\mathscr{U}^{\prime \prime}$ in $\mathscr{Y}(1)_{\mathbb{F}_{2}}$ contains the ordinary locus.

\section{ACKNOWLEDGEMENTS}

I thank Brian Conrad and Barry Mazur for helpful conversations and correspondence. I thank the referee for helpful comments and suggestions. I thank the Miller Institute for Basic Research in Science at the University of California Berkeley for its support. 


\section{REFERENCES}

AOV08 D. Abramovich, M. Olsson, and A. Vistoli, Tame stacks in positive characteristic, Ann. Inst. Fourier (Grenoble) 58 (2008), no. 4, 1057-1091; https://doi.org/10.5802/aif.2378.

Čes15 K. Česnavičius, A modular description of $\mathscr{X}_{0}(n), 2015$, arXiv:1511.07475.

Del75 P. Deligne, Courbes elliptiques: formulaire d'après J. Tate, Modular Functions of One Variable, IV (Proc. Internat. Summer School, Univ. Antwerp, Antwerp, 1972), Lecture Notes in Math., vol. 476 (Springer, Berlin, 1975), 53-73; https://doi.org/10.1007/BFb0097583.

DR73 P. Deligne and M. Rapoport, Les schémas de modules de courbes elliptiques, Modular Functions of One Variable, II (Proc. Internat. Summer School, Univ. Antwerp, Antwerp, 1972), Lecture Notes in Math., vol. 349 (Springer, Berlin, 1973), 143-316; https://doi.org/10.1007/ 978-3-540-37855-6_4.

EGAIV $_{2}$ A. Grothendieck and J. Dieudonné, Éléments de géométrie algébrique. IV. Étude locale des schémas et des morphismes de schémas. Seconde partie, Publ. Math. Inst. Hautes Études Sci. (1965), no. 24, 5-231; http://www.numdam.org/item?id=PMIHES_1965__24_5_0.

KM85 N. M. Katz and B. Mazur, Arithmetic moduli of elliptic curves, Ann. of Math. Stud., vol. 108 (Princeton Univ. Press, Princeton, NJ, 1985); https://doi.org/10.1515/9781400881710.

LM00 G. Laumon and L. Moret-Bailly, Champs algébriques, Ergeb. Math. Grenzgeb. (3), vol. 39 (Springer-Verlag, Berlin, 2000).

Rom05 M. Romagny, Group actions on stacks and applications, Michigan Math. J. 53 (2005), no. 1, 209-236; https://doi.org/10.1307/mmj/1114021093.

Ryd13 D. Rydh, Existence and properties of geometric quotients, J. Algebraic Geom. 22 (2013), no. 4, 629-669; https://doi.org/10.1090/S1056-3911-2013-00615-3.

Kęstutis Česnavičius kestutis@berkeley.edu

Department of Mathematics, University of California, Berkeley, CA 94720-3840, USA 